\title{
TOWARDS MULTISCALE FUNCTIONS: ENRICHING FINITE ELEMENT SPACES WITH LOCAL BUT NOT BUBBLE-LIKE FUNCTIONS
}

\author{
LEOPOLDO P. FRANCA, ALEXANDRE L. MADUREIRA, AND FREDERIC VALENTIN
}

\begin{abstract}
In this paper we propose a novel way, via finite elements to treat problems that can be singular perturbed, a reaction-diffusion equation in our case. We enrich the usual piecewise linear or bilinear finite element trial spaces with local solutions of the original problem, as in the Residual Free Bubble (RFB) setting, but do not require these functions to vanish on each element edge, a departure from the RFB paradigm. Such multiscale functions have an analytic expression, for triangles and rectangles. Bubbles are the choice for the test functions allowing static condensation, thus our method is of Petrov-Galerkin type. We perform several numerical validations which confirm the good performance of the method.
\end{abstract}

\section{Introduction}

It is well known that the standard finite element method based on piecewise polynomial approximations is unable to adequately model singularly perturbation equations (e.g., see [19] and references therein).

Previous works $[8,16,21,9]$ carried out more stable and accurate formulations based on stabilized methods for the reaction-diffusion model. These formulations are based on piecewise polynomials employed on modified variational formulations. These modifications are additional perturbation terms involving stability parameters and are functions of residuals of the governing differential equation.

Partial justification of these ideas were made possible by relating them to the Galerkin method using piecewise polynomials enriched with "bubble" functions, as illustrated in $[1$, $3,4]$. For the sake of simplicity, consider at this point bubble functions as continuous within each element and with zero value on the elements boundaries. The relation between these enriched Galerkin methods to stabilized ones is based on eliminating the bubble functions at the element level (a.k.a. static condensation), made possible due to the assumption of the zero value on the element boundary. This produces a stabilized-like formulation, in which

Key words and phrases. reaction-diffusion problem, multiscale finite element method, bubble function, boundary layer. 
the stability parameter is given by the shape of the bubble function, i.e., there is no ad hoc procedure to establish these parameters, other than selecting bubble functions.

To systematically treat various singularly perturbed problems, residual-free bubbles were introduced in $[6,10,11,12,13,14]$. These bubbles are produced by solving, exactly or not, differential equations at the element level, involving the differential operator of the problem. The right hand sides of these local problems are the residuals due to the polynomial part of the solution. The other ingredient is the requirement that the bubble part vanishes on element boundaries for second order problems.

It turns out that this construction for the reaction diffusion problem yields a poor approximation. Assuming the bubble part of the trial solution to be zero introduces inaccuracies across element edges. We wish to explore a possible avenue that builds on former ideas, without the zero boundary value restriction on elements, as follows:

(1) We let the test space to be enriched with residual-free bubble functions;

(2) We let the trial space to be derived from the previous construction with boundary values determined by local restriction of the governing differential operator.

Therefore we start out with a Petrov-Galerkin setting.

We keep the restriction of zero value on element boundary for the test space bubble functions, so that we can still use the static condensation argument. In this manner we are allowed to integrate by parts and get a differential equation for the trial enrichment, as before. Even more importantly, we keep the modification computable at the element level.

Now, we are in principle free to set the boundary condition for the trial enrichment. Towards this end, we use the restriction of the differential operator on the element edges and get ordinary differential equations that can be solved a priori. A related idea was proposed by $\mathrm{Hou}$ and $\mathrm{Wu}[17,18]$ for some multiscale examples. A numerical analysis for the present method is performed in [7].

The paper is organized as follows. In Section 2 our Petrov-Galerkin formulation is introduced. In Section 3 approximations of the solution for the trial enrichment at the element level are discussed, and next, in Section 4, we perform numerical tests.

\section{The Enriched Space Approach}

Let $\Omega$ be a bounded domain in $\mathbb{R}^{2}$ with polygonal boundary $\partial \Omega$. We consider $u \in H_{0}^{1}(\Omega)$ the solution of the reaction diffusion equation

$$
\mathcal{L} u:=-\varepsilon \Delta u+\sigma u=f \quad \text { in } \Omega, \quad u=0 \quad \text { on } \partial \Omega,
$$

where $\varepsilon$ and $\sigma$ are positive constants. We assume $f \in L^{2}(\Omega)$, thus (1) is well-posed. 
The usual weak formulation of problem (1) consists on finding $u \in H_{0}^{1}(\Omega)$ such that

$$
a(u, v)=(f, v), \quad \text { for all } v \text { in } H_{0}^{1}(\Omega),
$$

where the bilinear form $a: H_{0}^{1}(\Omega) \times H_{0}^{1}(\Omega) \rightarrow \mathbb{R}$ is given by

$$
a(u, v):=\varepsilon(\nabla u, \nabla v)+\sigma(u, v) .
$$

As usual $(\cdot, \cdot)_{D}$ denotes the inner product in $L^{2}(D)$ where $D$ is an open set of $\Omega$. To simplify the notation, we write $(\cdot, \cdot)$ when $D=\Omega$. The weak problem (2) is well-posed thanks to the coercivity of the bounded bilinear form $a(\cdot, \cdot)$ over $H_{0}^{1}(\Omega)$ and the Lax-Milgram Theorem.

Let $\mathcal{T}_{h}$ be a regular partition of domain $\Omega$ into elements $K$ (triangles or quadrangles) with boundary $\partial K$ such that

$$
\bar{\Omega}=\bigcup_{K \in \mathcal{T}_{h}} \bar{K}
$$

where the intersection of two elements is either a vertex, or an edge, or empty. We define $\mathcal{V}_{h}$ as the set of edges $Z$ belonging to $\mathcal{T}_{h}$, we denote by $h_{K}$ the diameter of $K \in \mathcal{T}_{h}$, and we set $h=\max _{K \in \mathcal{T}_{h}}\left\{h_{K}\right\}$.

The space of piecewise linear polynomials $\mathbb{P}^{1}(K)$ is used to approximate the exact solution. The same strategy is also valid for approximations in the bilinear space $\mathbb{Q}^{1}(K)$ as far as the elements $K \in \mathcal{T}_{h}$ are defined as affine transformation of the unit square. We denote the standard finite element space by

$$
V_{h}:=\left\{v_{h} \in H^{1}(\Omega) \mid v_{\left.h\right|_{K}} \in \mathbb{P}^{1}(K) \text { for all } \mathrm{K} \in \mathcal{T}_{h}\right\}
$$

and

$$
V_{h}^{0}:=V_{h} \cap H_{0}^{1}(\Omega)
$$

The standard Galerkin scheme associated to the continuous problem reads: find $u_{g} \in V_{h}^{0}$ such that

$$
a\left(u_{g}, v_{1}\right)=\left(f, v_{1}\right), \quad \text { for all } v_{1} \text { in } V_{h}^{0}
$$

It is well known that the Galerkin method (5) is unable to approximate the boundary layers present in the solution if $\varepsilon \ll \sigma h^{2}$. Therefore, we are interested in finding a finite element discretization for (2) that is stable and coarse mesh accurate for all $\varepsilon$ and $\sigma$. We use the approach of enriching the finite element space. The idea is to add special functions, also called multiscale functions, to the usual polynomial spaces to stabilize and improve the accuracy of 
the Galerkin method. Henceforth, we propose a Petrov-Galerkin approach wherein we keep the space of test functions as polynomial plus bubbles, i.e.,

$$
V_{h}^{0} \oplus H_{0}^{1}\left(\mathcal{T}_{h}\right)
$$

where $H_{0}^{1}\left(\mathcal{T}_{h}\right)$ and $H^{1}\left(\mathcal{T}_{h}\right)$ are the spaces of functions on $\Omega$ whose restriction to each element $K$ belongs to $H_{0}^{1}(K)$ and $H^{1}(K)$, respectively. Furthermore, we denote by $E_{h}$ a subspace of $H^{1}\left(\mathcal{T}_{h}\right)$, called multiscale space, such that $E_{h} \cap V_{h}^{0}=\{0\}$.

We introduce the trial subspace $U_{h}$ of $H^{1}\left(\mathcal{T}_{h}\right)$ defined by

$$
U_{h}:=V_{h}^{0} \oplus E_{h}
$$

thus an element $v_{h}$ of $U_{h}$ may be uniquely written as

$$
v_{h}:=v_{1}+v_{e}
$$

where $v_{1} \in V_{h}^{0}$ and $v_{e} \in E_{h}$. Therefore, our approximation of the exact solution in the enriched space (6), is defined by the solution of the following Petrov-Galerkin problem: find $u_{h} \in U_{h}$ such that

$$
a_{h}\left(u_{h}, v_{h}\right)=\left(f, v_{h}\right), \quad \text { for all } v_{h} \in V_{h}^{0} \oplus H_{0}^{1}\left(\mathcal{T}_{h}\right)
$$

where

$$
a_{h}(u, v):=\sum_{K \in \mathcal{T}_{h}} a(u, v)_{K}
$$

and

$$
a(u, v)_{K}:=\varepsilon(\nabla u, \nabla v)_{K}+\sigma(u, v)_{K}
$$

From (7) we immediately have that the corresponding $u_{h} \in U_{h}$ satisfies

$$
\begin{gathered}
a_{h}\left(u_{h}, v_{1}\right)=\left(f, v_{1}\right) \quad \text { for all } v_{1} \in V_{h}^{0}, \\
a\left(u_{h}, v_{b}^{K}\right)_{K}=\left(f, v_{b}^{K}\right)_{K} \quad \text { for all } v_{b}^{K} \in H_{0}^{1}(K) .
\end{gathered}
$$

The well-posedness of (7) is discussed in [7]. Integrating (9) by parts, we immediately have that the enriched part of the solution $u_{h}$, denoted by $u_{e} \in E_{h}$, is the strong solution of the local problem

$$
\mathcal{L} u_{e}=f-\mathcal{L} u_{1} \quad \text { in each } K \in \mathcal{T}_{h}
$$

In order to have (10) well-posed we must set boundary conditions on $\partial K$. We perform that by introducing the operator $\mathcal{B}_{K}: L^{2}(\partial K) \rightarrow H^{1}(\partial K)$ defined in the following way: 
given $w_{1} \in L^{2}(\partial K)$ we associate $w_{e}=\mathcal{B}_{K} w_{1} \in H^{1}(\partial K)$ such that

$$
\mathcal{L}_{\partial K} w_{e}:=-\varepsilon \partial_{s s} w_{e}+\bar{\sigma} w_{e}=w_{1} \text { and } w_{e}=0 \text { at the nodes. }
$$

The coefficient $\bar{\sigma}$ is set as a positive constant which can depend on $|K|$. Such dependence will be specified later (see equation (35) for the triangle element case), and we denote by $s$ a variable that parametrize $\partial K$ by arc-length. We point out that $(11)$ is well-posed. A similar boundary condition was used in Hou et al. for elliptic problems with oscillating coefficients (see $[17,18])$.

Now, let $\mathcal{M}_{K}: H^{1}(K) \rightarrow H^{2}(K)$ be the linear operator defined as follows: given $v_{1} \in$ $H^{1}(K)$ let $v_{e}=\mathcal{M}_{K} v_{1} \in H^{2}(K)$ be the solution of the problem

$$
\begin{gathered}
\mathcal{L} v_{e}=v_{1} \text { in } K, \\
v_{e}=\mathcal{B}_{K}\left(\frac{\bar{\sigma}}{\sigma} v_{1}\right) \text { on } \partial K,
\end{gathered}
$$

where $\mathcal{B}_{K}$ is the linear local operator defined in (11).

Problem (12), (13) is clearly well-posed in each $K \in \mathcal{T}_{h}$. Therefore, from (10) we impose

$$
u_{e}=\mathcal{M}_{K}\left(f-\mathcal{L} u_{1}\right),
$$

and from now on, we assume that $f \in V_{h}$.

Remark 1. The standard enriched strategy with bubble-like functions, as the Residual-FreeBubble (RFB) approach $[3,5,6]$, is similar to (14) where $u_{e}$ vanishes on all $\partial K \in \mathcal{V}_{h}$. In both cases, we have the same local operator. However, thanks to the boundary condition (11) we will be able to obtain analytic solutions.

Inspired by (14) we define the multiscale finite dimensional space $E_{h}$ by

$$
E_{h}:=\left\{v_{e} \in H^{1}\left(\mathcal{T}_{h}\right) \mid v_{\left.e\right|_{K}}=\mathcal{M}_{K} v_{1} \text { for all } v_{1} \in V_{h}\right\},
$$

and it follows by construction and by (8) that (7) is equivalent to the finite dimensional problem: find $u_{1} \in V_{h}^{0}$ such that

$$
a_{h}\left(\left(\mathcal{I}-\mathcal{M}_{K} \mathcal{L}\right) u_{1}, v_{1}\right)=\left(f, v_{1}\right)-a_{h}\left(\mathcal{M}_{K} f, v_{1}\right) \quad \text { for all } v_{1} \in V_{h}^{0},
$$

where $\mathcal{I}$ is the identity operator. The variational formulation (15) can be seen as the standard Galerkin method with the modified bilinear and linear forms

$$
a\left(u_{1}, v_{1}\right)-a_{h}\left(\mathcal{M}_{K} u_{1}, \sigma v_{1}\right)=\left(f, v_{1}\right)-a_{h}\left(\mathcal{M}_{K} f, v_{1}\right) \text { for all } v_{1} \in V_{h}^{0},
$$

where we have used that $u_{1}$ is piecewise linear. 
Remark 2. The space $E_{h}$ is a finite dimensional space and $\operatorname{dim}\left(E_{h}\right)=\operatorname{dim}\left(V_{h}\right)$. We note from (13) that the functions belonging to $E_{h}$ may be a priori discontinuous across the edges of triangles. The continuity is enforced only at the nodes of the triangulation. Therefore, the method is nonconforming.

Remark 3. Concerning stabilized finite element method theory, it is interesting to observe some analogies between enriched approach and the unusual method proposed in [15]. The bilinear form (15) corresponds to the Residual-Free-Bubble one [20] in the case that $u_{e}$ vanishes on the boundary of each element. We recall that the unusual method reads: find $u_{1}^{s} \in V_{h}^{0}$ such that

$$
a_{s}\left(u_{1}^{s}, v_{1}\right)=f_{s}\left(v_{1}\right) \quad \text { for all } v_{1} \in V_{h}^{0},
$$

where the bilinear form $a_{s}: V_{h}^{0} \times V_{h}^{0} \rightarrow \mathbb{R}$ reads

$$
a_{s}(u, v):=a(u, v)-\sum_{K \in T_{h}}\left(\tau_{K} \mathcal{L} u, \sigma v\right)_{K}
$$

and the linear form $f_{s}: V_{h}^{0} \rightarrow \mathbb{R}$ is defined by

$$
f_{s}(v):=(f, v)-\sum_{K \in T_{h}}\left(\tau_{K} f, \sigma v\right)_{K} .
$$

The stabilization parameter $\tau_{K}$ is a piecewise constant function defined by

$$
\tau_{K}=\frac{h_{K}^{2}}{\sigma h_{K}^{2} \max \left\{1, P e_{K}\right\}+6 \varepsilon},
$$

where $P e_{K}$ is the Peclet number

$$
P e_{K}=\frac{6 \varepsilon}{\sigma h_{K}^{2}} .
$$

It is possible to show that enriched methods, particularly the RFB formulation, is equivalent to (3) as $\mathcal{M}_{K}=\tau_{K} \mathcal{I}$ (see [9] and [2] for the case of Stokes operator). With respect to the proposed enriched approach, we believe that it is equivalent to the unusual method, but such equivalence in not trivial. This shall be investigated in a future work.

\section{LOCAL PROBLEM}

3.1. Corresponding discrete formulation. The resolution of the weak problem (15) requires the resolution of the local problem (14) for each $K \in \mathcal{T}_{h}$, i.e, we need to find $u_{e} \in E_{h}$ 
such that

$$
\begin{gathered}
\mathcal{L} u_{e}=f-\sigma u_{1} \text { in } K, \\
u_{e}=\mathcal{B}_{K}\left(\frac{\bar{\sigma}}{\sigma} f-\bar{\sigma} u_{1}\right) \text { on } \partial K,
\end{gathered}
$$

where the operator $\mathcal{B}_{K}$ is defined in (11). Let us rewrite (17), (18) in terms of basis functions. We assume that

$$
E_{h}=\operatorname{span}\left\{\phi_{i}\right\}_{i \in I} \text { and } V_{h}=\operatorname{span}\left\{\psi_{i}\right\}_{i \in I},
$$

where $\psi_{i}$ are the usual hat functions. Then, $f$ and $u_{1}$ are given by

$$
u_{1}=\sum_{i \in I_{0}} \psi_{i} u_{i}, \quad f=\sum_{j \in I} \psi_{j} f_{j},
$$

where $u_{i}, i \in I_{0}$, and $f_{j}, j \in I$, are the nodal values of $u$ and $f$, respectively. Here $I$ and $I_{0}$ are the set of indexes of total and internal nodal points, respectively. It follows from (17), (18), and from the linearity of the operators $\mathcal{L}$ and $\mathcal{L}_{\partial K}$ that

$$
u_{e}=\sum_{i \in I_{0}} \phi_{i} u_{i}-\sum_{i \in I} \phi_{i} \frac{f_{i}}{\sigma}
$$

where the basis functions $\phi_{i} \in E_{h}, i \in I$, satisfy

$$
\begin{aligned}
\mathcal{L} \phi_{i} & =-\sigma \psi_{i} \quad \text { in } K, \\
\phi_{i} & =\mu_{i} \quad \text { on } \partial K,
\end{aligned}
$$

for all $K \in \mathcal{T}_{h}$. From (18) and (20), $\mu_{i} \in H^{1}(\partial K)$ is the solution of the boundary value problem

$$
\mathcal{L}_{\partial K} \mu_{i}=-\bar{\sigma} \psi_{i} \text { in } \partial K \text { and } \mu_{i}=0 \text { at the nodes, }
$$

on each edge belonging to $\partial K \in \mathcal{V}_{h}$. Now, let $\lambda_{j} \in U_{h}$ be defined by

$$
\lambda_{j}:=\psi_{j}+\phi_{j}=\left(\mathcal{I}-\sigma \mathcal{M}_{K}\right) \psi_{j} \quad \text { for all } j \in I .
$$

Thus the discrete version of the weak formulation (15) reads

$$
\sum_{j \in I_{0}} a\left(\lambda_{j}, \psi_{i}\right) u_{j}=\sum_{j \in I}\left[\left(\lambda_{j}, \psi_{i}\right)-\frac{\varepsilon}{\sigma}\left(\nabla \psi_{j}, \nabla \psi_{i}\right)+\frac{\varepsilon}{\sigma}\left(\nabla \lambda_{j}, \nabla \psi_{i}\right)\right] f_{j} \quad \text { for all } i \in I_{0},
$$

or equivalently

$$
\sum_{j \in I_{0}} a\left(\lambda_{j}, \psi_{i}\right) u_{j}=\sum_{j \in I}\left[a\left(\lambda_{j}, \psi_{i}\right)-\varepsilon\left(\nabla \psi_{j}, \nabla \psi_{i}\right)\right] \frac{f_{j}}{\sigma} \quad \text { for all } i \in I_{0}
$$

In order to solve the weak problem (25) we have to calculate $\phi_{i} \in E_{h}, i \in I$, from (21)-(23) what is done analytically in the next subsection. A typical solution of (21)-(23) is shown in 
Figure 1 wherein we set $\sigma=1$ and $\varepsilon=10^{-3}$. Note that the support of $\phi_{i}$ coincide with the support of the piecewise linear function $\psi_{i}$.
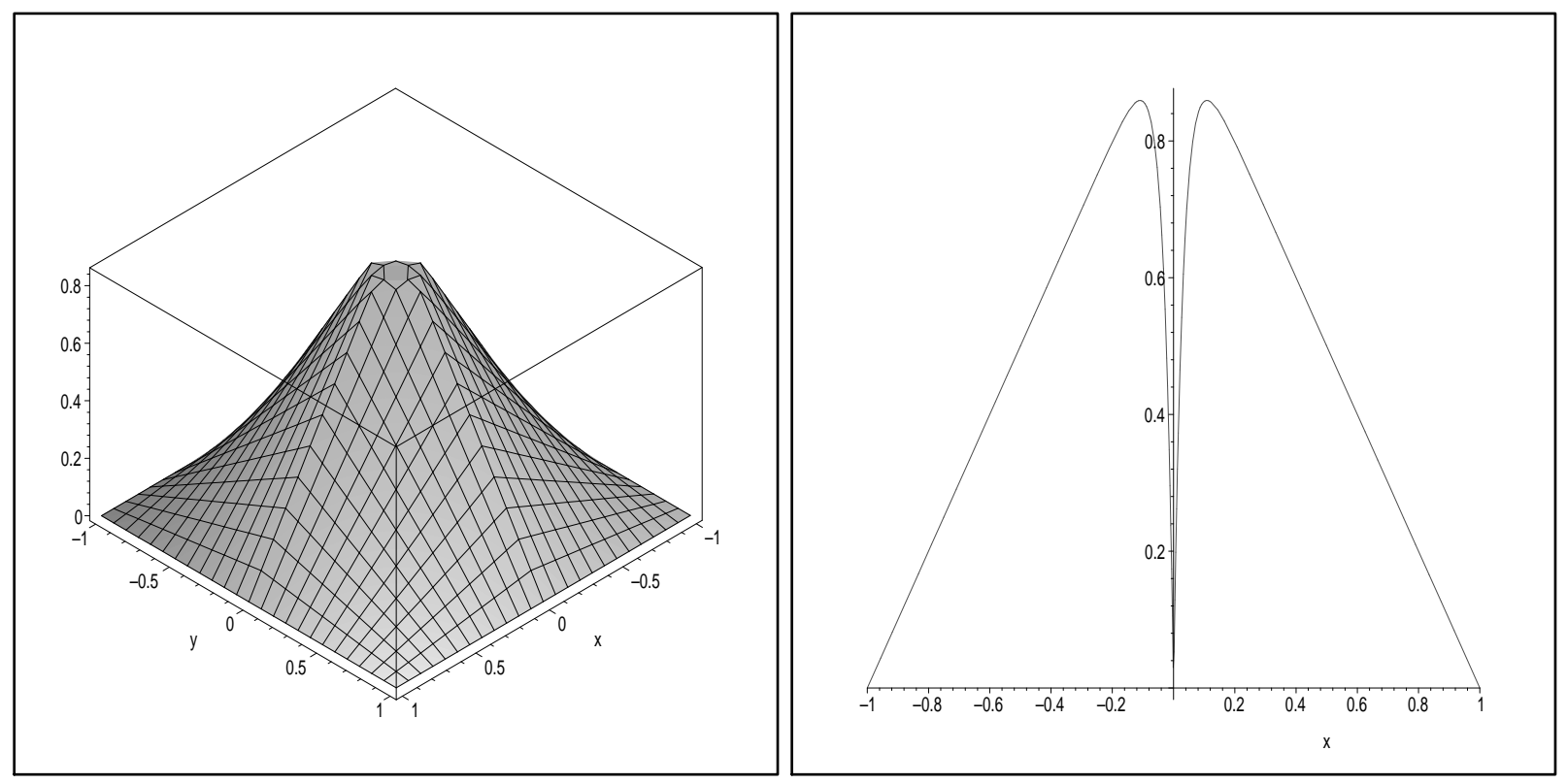

Figure 1. An enriching function $-\phi_{i}$ (left) and its cross section at $x=0$ (right).

Remark 4. In the particular case where $f$ is assumed to be constant, the weak formulation (25) becomes

$$
\sum_{j \in I_{0}} a\left(\lambda_{j}, \psi_{i}\right) u_{j}=\sum_{j \in I} a\left(\lambda_{j}, \psi_{i}\right) \frac{f_{j}}{\sigma} \quad \text { for all } i \in I_{0} .
$$

Numerical experiments indicate that the modified scheme type

$$
\sum_{j \in I_{0}} a\left(\lambda_{j}, \psi_{i}\right) u_{j}=\sum_{j \in I}\left(\lambda_{j}, \psi_{i}\right) \frac{f_{j}}{\sigma} \quad \text { for all } i \in I_{0},
$$

yields accurate numerical approximations. This mass lumping trick is, nonetheless, contrary to our general philosophy of deriving the formulation through a sound formalism. Thus, we do not advocate this approach.

3.2. Solving the local problem. The present method requires solving the local problems (21)-(23). It is convenient to present such problem in terms of the unknown $\lambda_{i}, i \in I$, introduce in (24). Let $\rho_{i}$ be the restriction of $\lambda_{i}$ to $\partial K$, i.e.,

$$
\rho_{i}:=\psi_{\left.i\right|_{\partial K}}+\phi_{\left.i\right|_{\partial K}}=\psi_{\left.i\right|_{\partial K}}+\mu_{i} \quad \text { for all } i \in I \text {. }
$$


Note from $(23)$ the function $\rho_{i}, i \in I$, satisfies the ordinary differential problem

$$
\mathcal{L}_{\partial K} \rho_{i}=0 \text { on } \partial K \text { and } \rho_{i}=\psi_{i} \text { at the nodes, }
$$

on each edge belonging to $\partial K \in \mathcal{V}_{h}$. Hence, from the definitions (24) and (28), the function $\lambda_{i}, i \in I$, satisfies

$$
\begin{aligned}
& \mathcal{L} \lambda_{i}=0 \quad \text { in } K, \\
& \lambda_{i}=\rho_{i} \quad \text { on } \partial K,
\end{aligned}
$$

where $\rho_{i}$ is the solution of (29). Now, we present analytical solutions of problem (30), (31) when $K$ is either a triangle or a rectangle.

3.2.1. Bilinear shape functions. Consider a quadrilateral straight mesh. Thanks to the nonhomogenous boundary conditions (29) and choosing $\bar{\sigma}=2 \sigma$, it is quite easy to calculate $\lambda_{i}$ for $i \in I$. We observe that the method becomes conform since we impose continuity of shape functions on the boundary $\partial K$. Consider a rectangle $K$ with vertexes $1, \ldots, 4$ at $(0,0)$, $\left(h_{x}, 0\right),\left(h_{x}, h_{y}\right),\left(0, h_{y}\right)$.

Without loss of generality, we compute $\lambda_{1}$ solution of (30), (31). Since the bilinear function $\psi_{1}$ can be written as $\psi_{1}(x, y)=\psi_{x}^{1}(x) \psi_{y}^{1}(y)$, then on $y=0$, we have that

$$
\begin{gathered}
-\varepsilon \frac{\partial^{2} \lambda_{1}}{\partial x^{2}}+2 \sigma \lambda_{1}=0 \quad \text { for } x \text { in }\left(0, h_{x}\right), \\
\lambda_{1}(0,0)=1, \quad \lambda_{1}\left(h_{x}, 0\right)=0 .
\end{gathered}
$$

Hence,

$$
\lambda_{1}(x, 0)=\lambda_{x}^{1}(x):=\frac{\sinh \left(\sqrt{\frac{\sigma}{2 \varepsilon}} h_{x} \psi_{x}^{1}(x)\right)}{\sinh \left(\sqrt{\frac{\sigma}{2 \varepsilon} h_{x}}\right)} .
$$

Similarly,

$$
\lambda_{1}(0, y)=\lambda_{y}^{1}(y):=\frac{\sinh \left(\sqrt{\frac{\sigma}{2 \varepsilon}} h_{y} \psi_{y}^{1}(y)\right)}{\sinh \left(\sqrt{\frac{\sigma}{2 \varepsilon} h_{y}}\right)}, \quad \text { and } \lambda_{1}\left(h_{x}, y\right)=\lambda_{1}\left(x, h_{y}\right)=0 .
$$

We remark that $\lambda_{1}(x, y)=\lambda_{x}^{1}(x) \lambda_{y}^{1}(y)$ satisfies (30), (31) exactly. Then

$$
\lambda_{1}(x, y)=\frac{\sinh \left(\sqrt{\frac{\sigma}{2 \varepsilon}} h_{x} \psi_{x}^{1}(x)\right) \sinh \left(\sqrt{\frac{\sigma}{2 \varepsilon}} h_{y} \psi_{y}^{1}(y)\right)}{\sinh \left(\sqrt{\frac{\sigma}{2 \varepsilon} h_{x}}\right) \sinh \left(\sqrt{\frac{\sigma}{2 \varepsilon} h_{y}}\right)} .
$$

The basis functions $\lambda_{j}, j=2, . .4$, are immediately obtained from $\lambda_{1}$ by simply changing variables. If we take a particular node $l \in I$, and look at all elements connected to this node, then the equation (34) can be used to illustrate the nodal shape functions $\lambda_{l}$. Fixing $\sigma=1$, we obtain for $\varepsilon=1,10^{-1}, 10^{-3}$, the shape functions $\lambda_{l}$, depicted in Figures 2 and 3 . Note 
that as $\varepsilon$ approaches zero, the usual pyramid is squeezed in its domain of influence in the neighborhood around node $l$.

3.2.2. Linear shape functions. Consider now a regular triangular mesh. Let $K$ be an element of the triangulation $\mathcal{T}_{h}$, and $Z$ an edge of its boundary $\partial K$. We explicit the dependence of coefficients $\bar{\sigma}$ in terms of the shape of elements $K$ by setting

$$
\bar{\sigma}:=\frac{\sigma}{\gamma_{K}^{i}|Z|^{2}} \quad \text { for all } i \in I
$$

where the positive constant $\gamma_{K}^{i}$ is defined by

$$
\gamma_{K}^{i}=\left(\left.\frac{\partial \psi_{i}}{\partial x}\right|_{K}\right)^{2}+\left(\left.\frac{\partial \psi_{i}}{\partial y}\right|_{K}\right)^{2} \quad \text { for all } i \in I
$$

Remark 5. We can identify the constant introduce in (36) in terms of the shape of $K$ as

$$
\gamma_{K}^{i}=\frac{|\bar{Z}|^{2}}{4|K|^{2}}
$$

where $\bar{Z}$ denotes the corresponding edge of $K$ opposed to the node $i$. We note that $\gamma_{K}^{i}$ is of order $h_{K}^{-2}$ for all $i \in I$ and $K \in \mathcal{T}_{h}$.

Thanks to the definitions (35) and (36) we are able to compute the analytical solution of (30), (31). Indeed, it is straightforward to check that

$$
\lambda_{i}(x, y)=\frac{\sinh \left(\sqrt{\frac{\sigma}{\varepsilon \gamma_{K}^{i}}} \psi_{i}(x, y)\right)}{\sinh \left(\sqrt{\frac{\sigma}{\varepsilon \gamma_{K}^{i}}}\right)} \text { for all } i \in I
$$

satisfies the boundary value problem (30), (31).

Remark 6. The enriched basis functions (37) are discontinuous across element edges since $\gamma_{K}^{i}$ varies a priori in each $K \in \mathcal{T}_{h}$. Therefore as we have already pointed out, the present method is nonconforming in general, but we can recover the continuity and the conformity in the case of the value of $\gamma_{K}^{i}$ is the same for all $K \in \mathcal{T}_{h}$. 

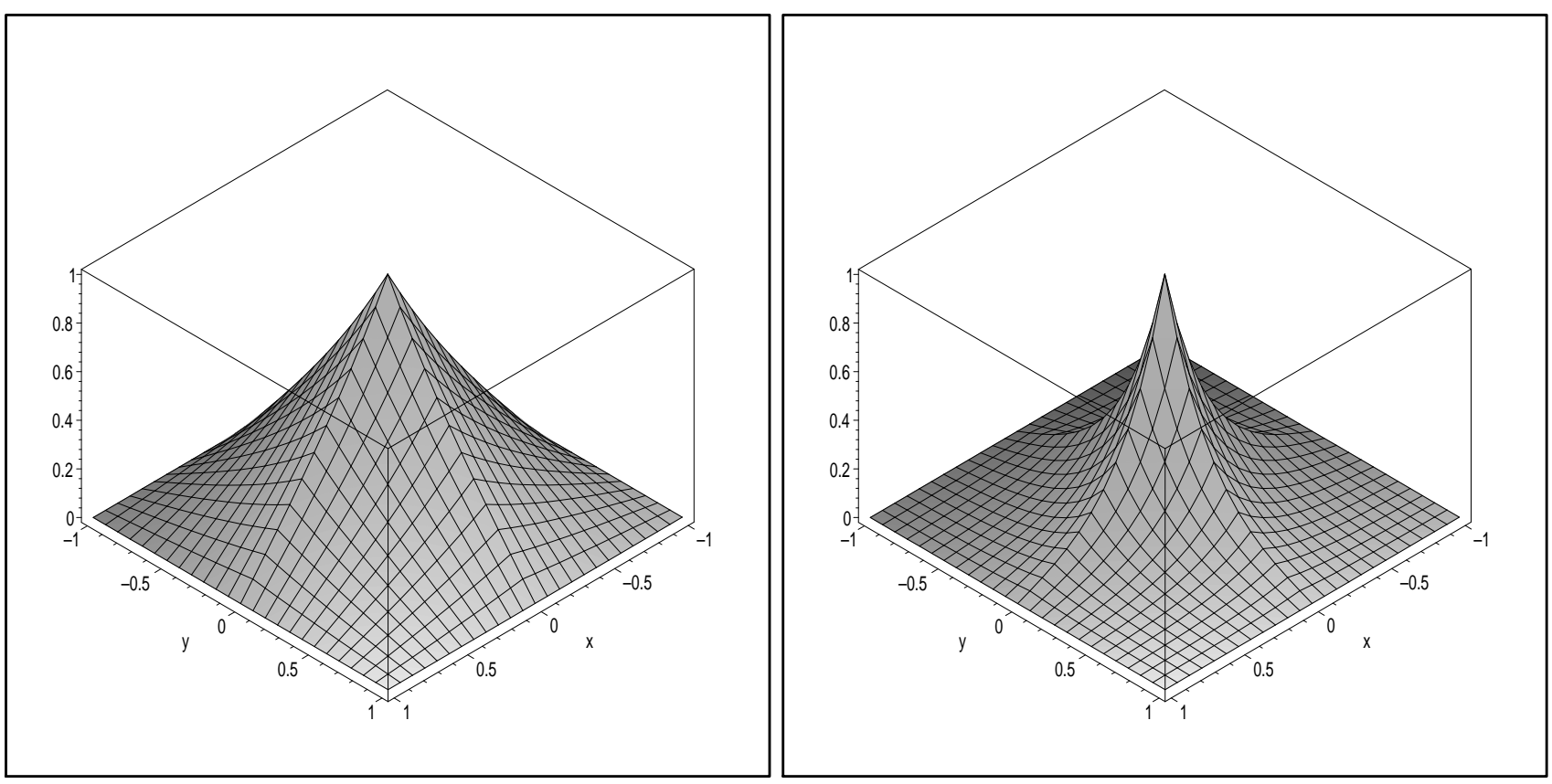

Figure 2. The function $\lambda_{l}$ for $\varepsilon=1$ (left) and $\varepsilon=10^{-2}$ (right).

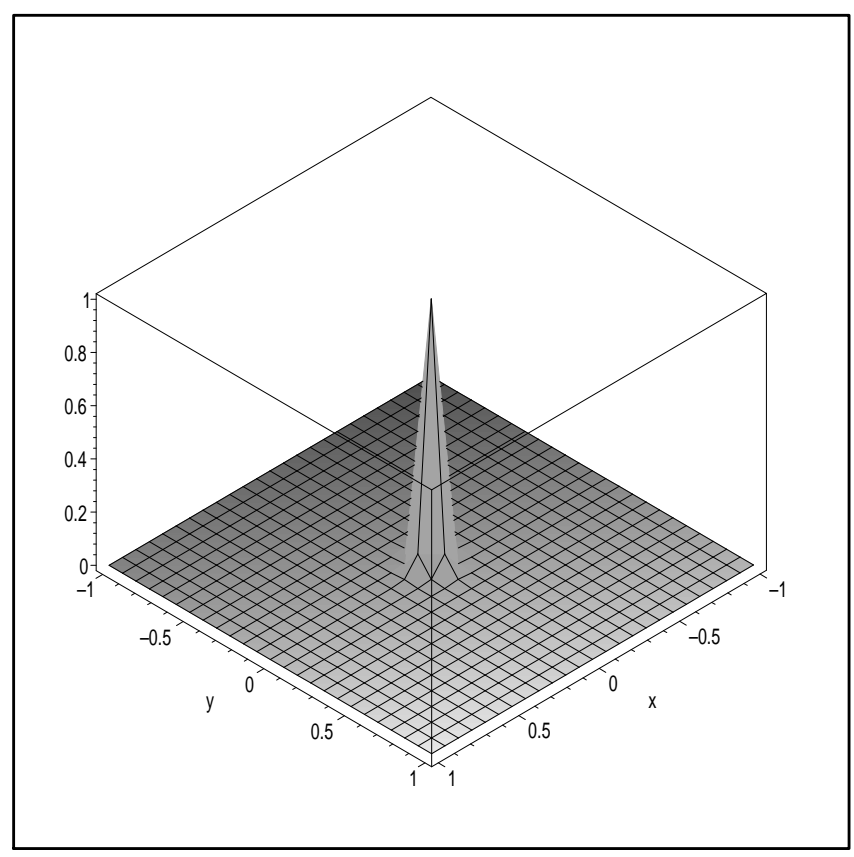

Figure 3. The function $\lambda_{l}$ for $\varepsilon=10^{-3}$. 


\section{Numerical Results}

4.1. Source problem. Let us first consider the unit source problem $(f=1)$ defined on the unit square depicted in Figure 4, subject to a homogeneous boundary condition.

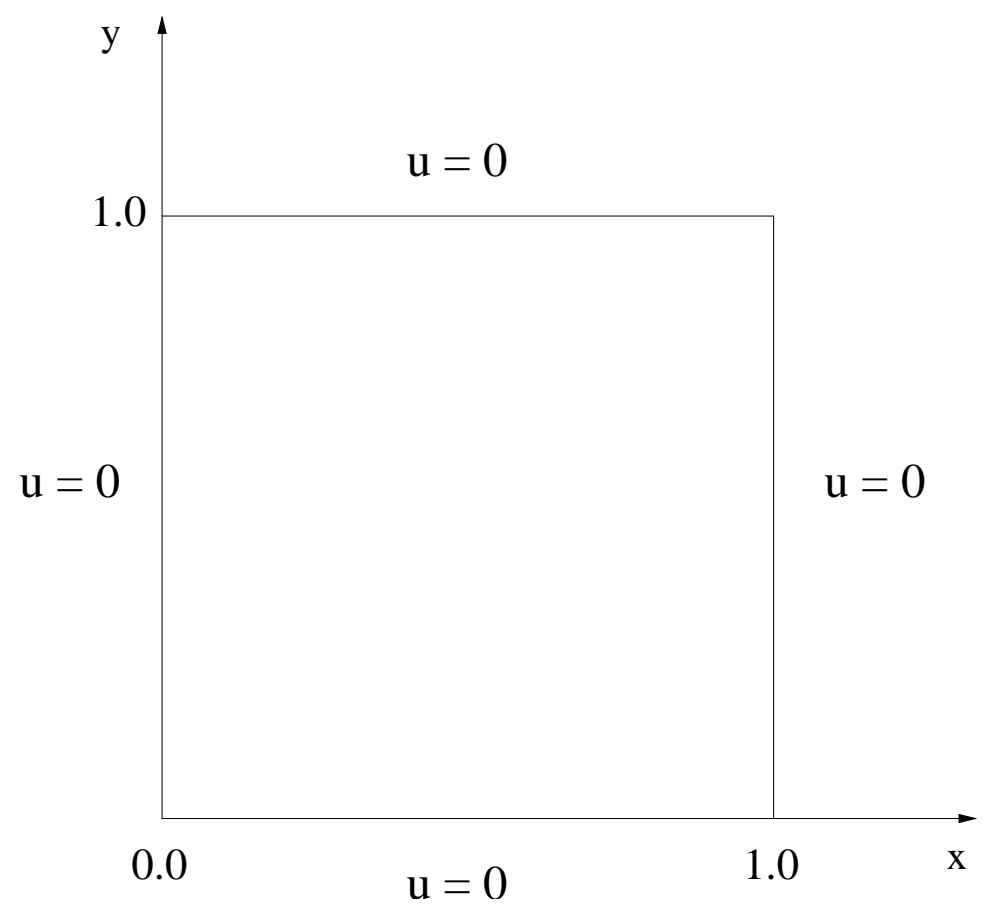

Figure 4. Problem statement.

For a fixed $\sigma=1$ and small $\varepsilon$, boundary layers appear close to the domain boundary. Figures 5, 6 shows the solutions of four different methods, for $\varepsilon=10^{-6}$. The unusual stabilized method and the current method perform better than the other two methods. For $\varepsilon=10^{-3}$ and $\varepsilon=1$, all methods have comparable performance, see Figures 7,8 . 
GALERKIN METHOD

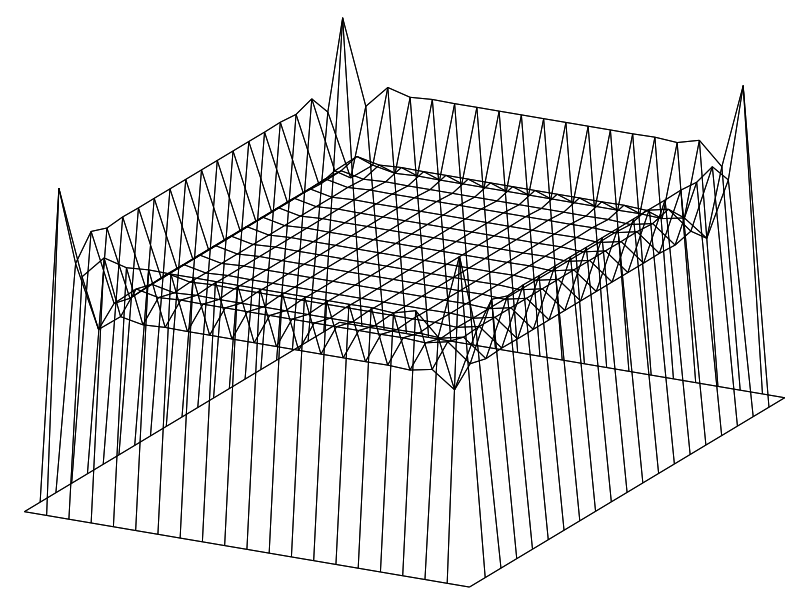

UNUSUAL METHOD

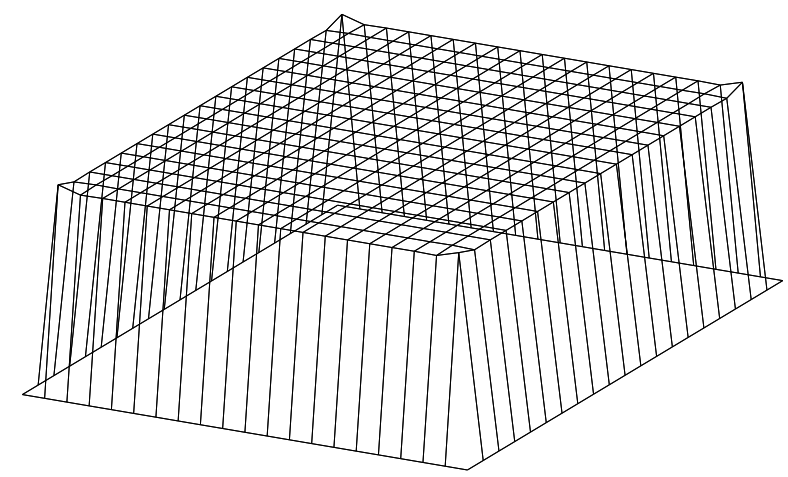

RFB METHOD

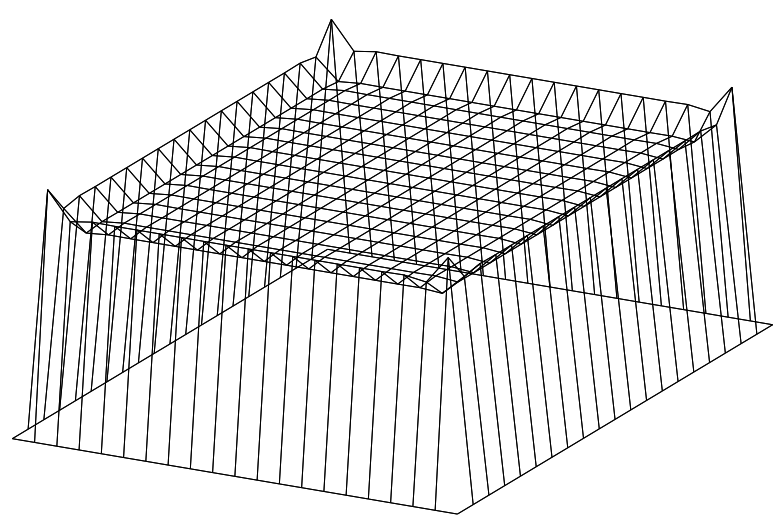

NEW ENRICHED METHOD\%anticlipinit

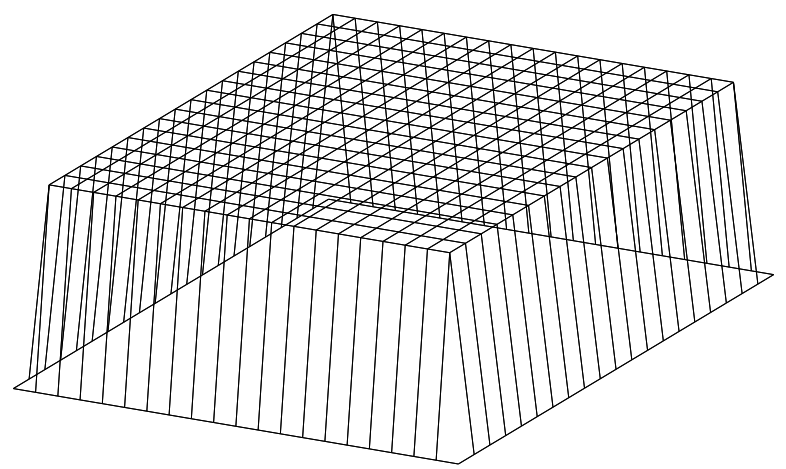

Figure 5. Comparison among Galerkin, Residual Free Bubble, unusual and the enriched method for $\varepsilon=10^{-6}$. 


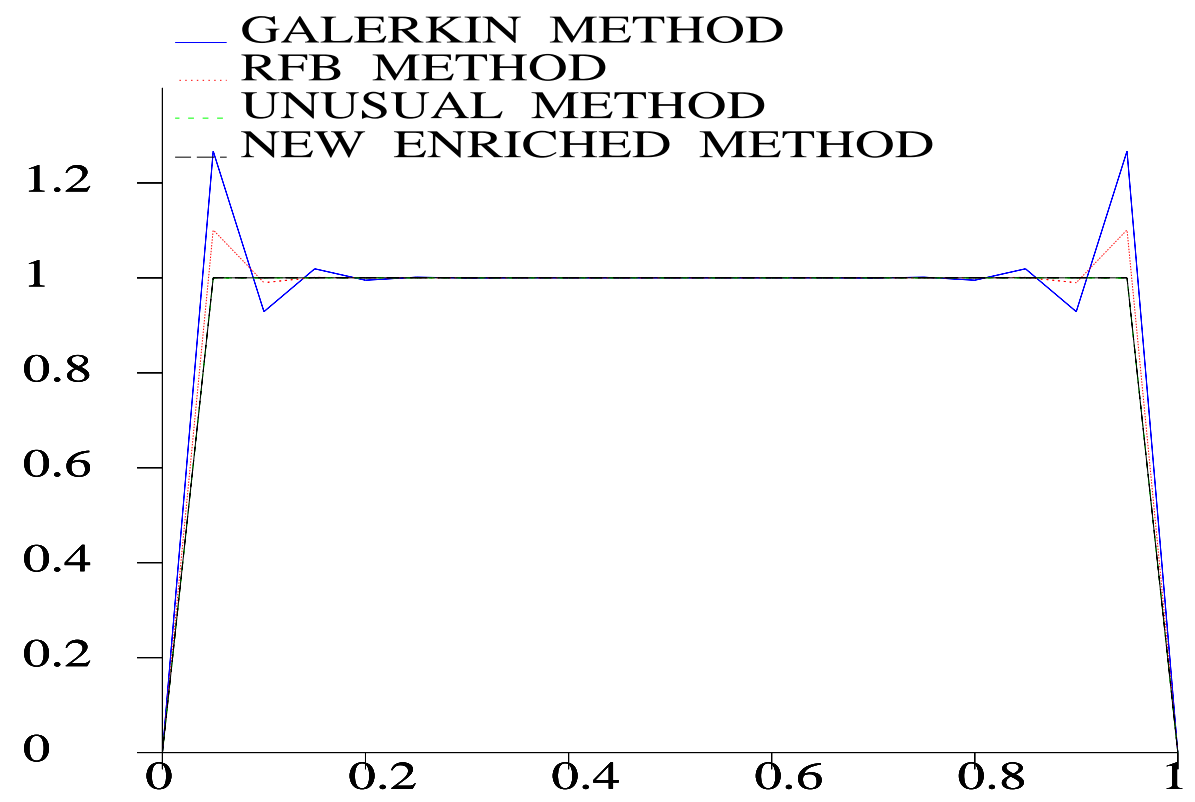

Figure 6. Profile of solutions at $x=0.5\left(\varepsilon=10^{-6}\right)$.

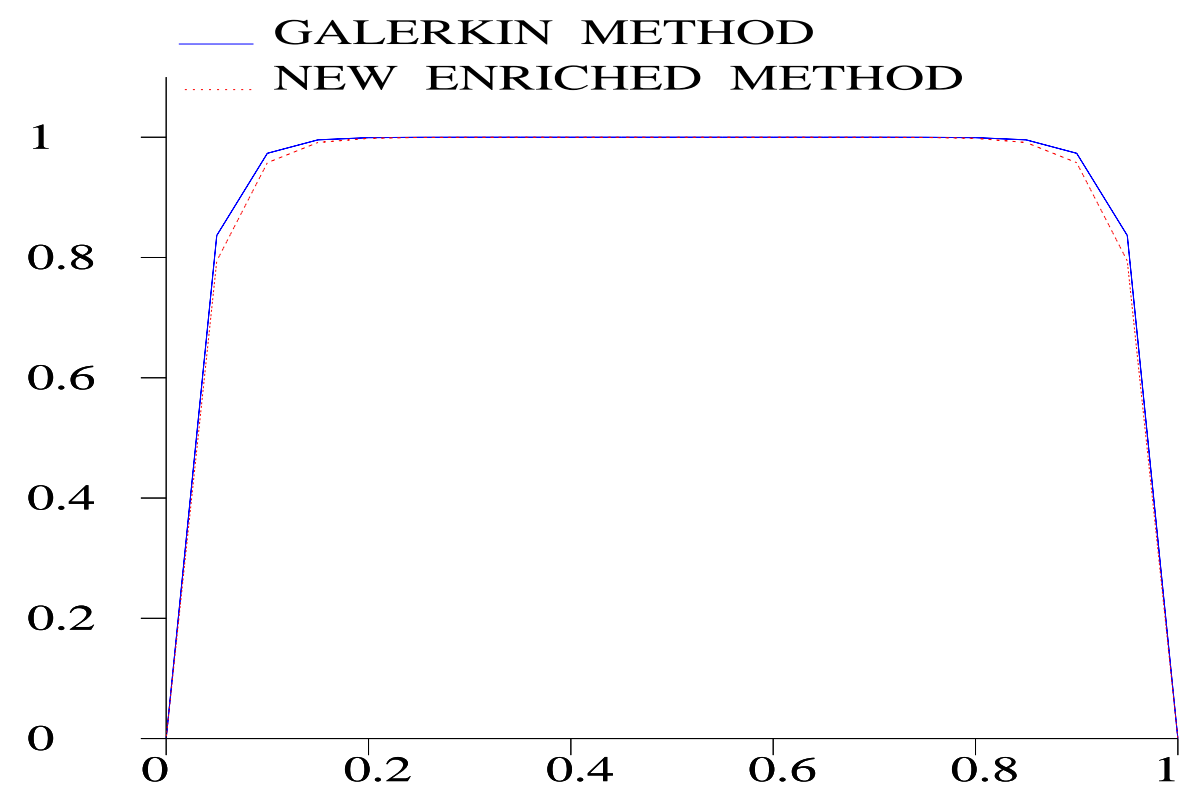

Figure 7. Profile of solutions at $x=0.5\left(\varepsilon=10^{-3}\right)$. 


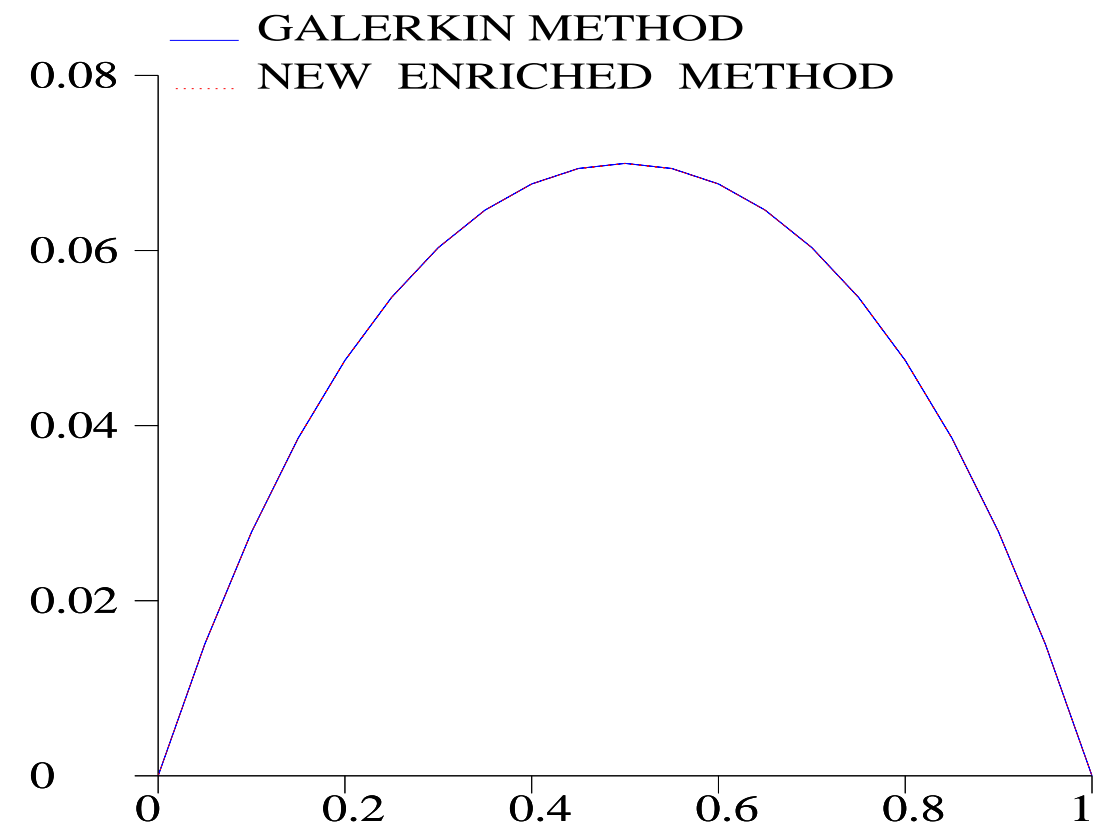

Figure 8. Profile of solutions at $x=0.5(\varepsilon=1)$.

4.2. Boundary layer problem using refined mesh. Here we consider a rectangular domain with nontrivial boundary conditions, as depicted in Figure 9. We assume that $f=0$, and the domain is discretized using a refined mesh in part of the domain.

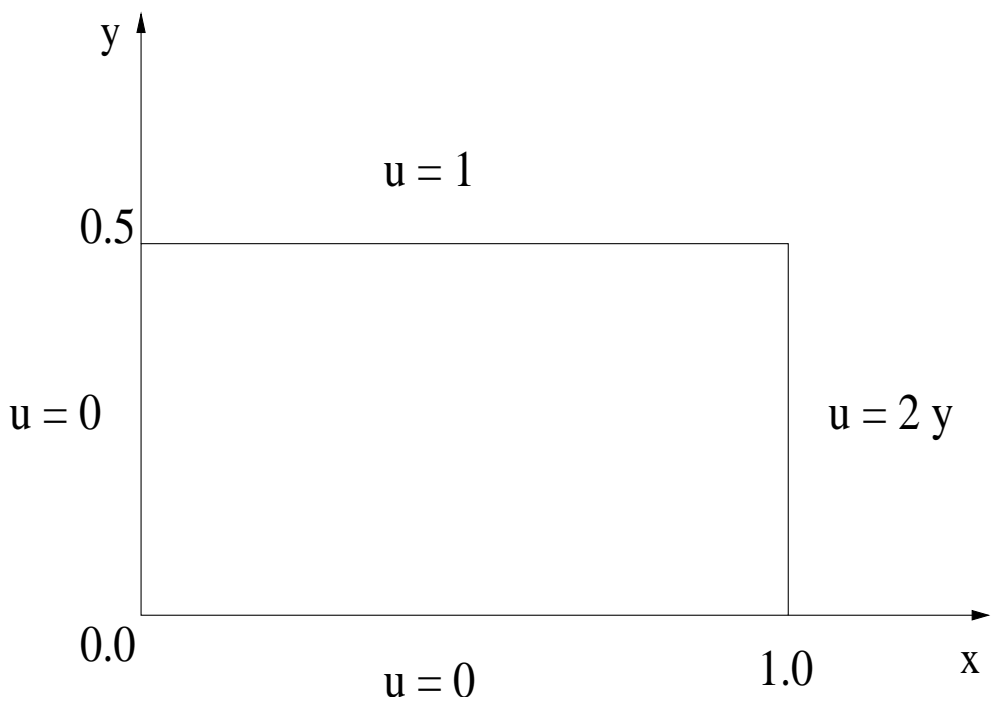

Figure 9. Problem statement. 
Again for this problem we have the onset of boundary layers that causes spurious oscillations in the numerical solutions. We computed the solution with different methods for $\sigma=1$, and $\varepsilon=10^{-6}$. Figures 10, 11, 12 show the good performance of the current method, while all other methods suffer from spurious oscillations. Finally, Figure 13 illustrate that for large $\varepsilon$, we recover the usual Galerkin method.
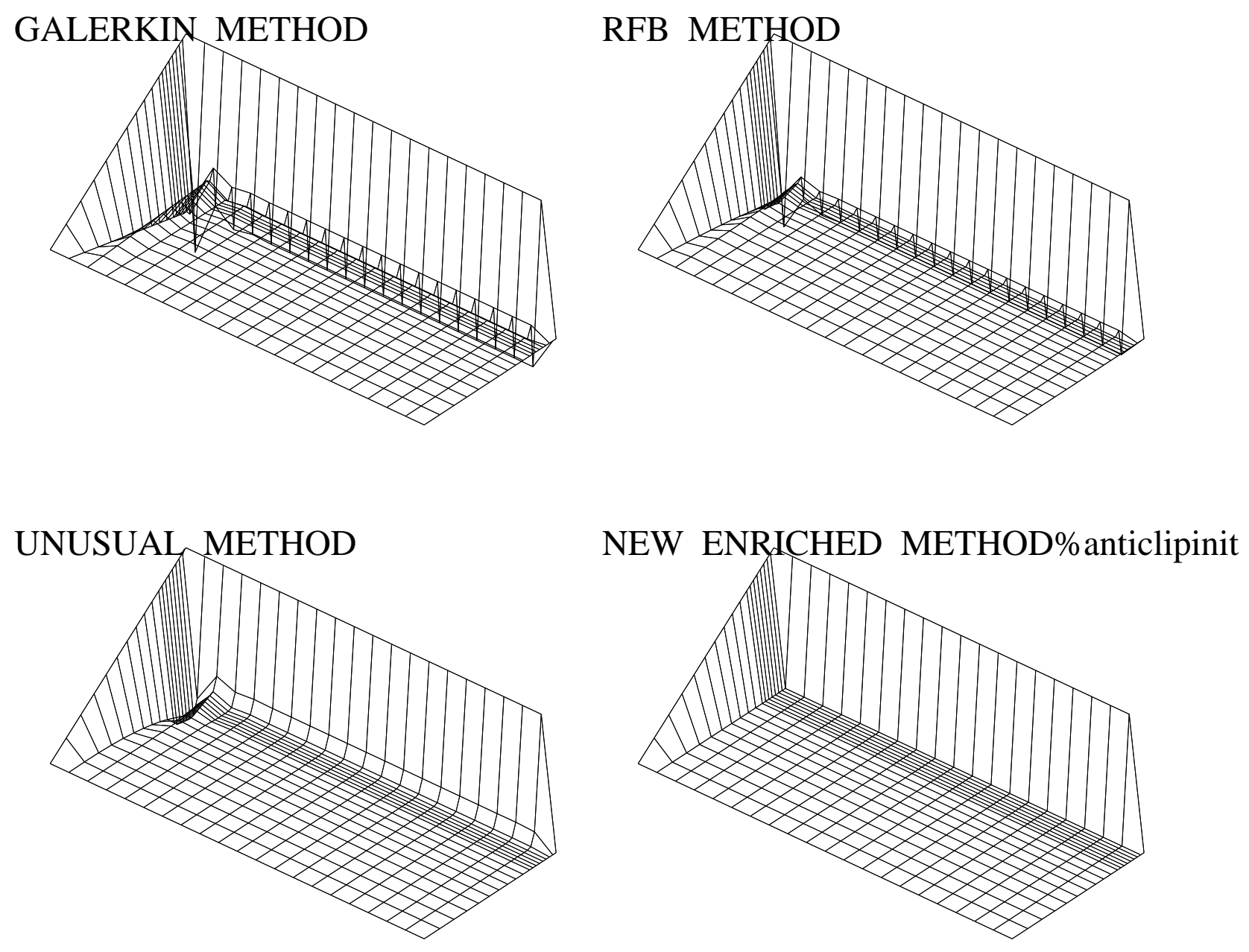

FiguRE 10. Comparison between solutions obtained by different methods $\left(\varepsilon=10^{-6}\right)$. 


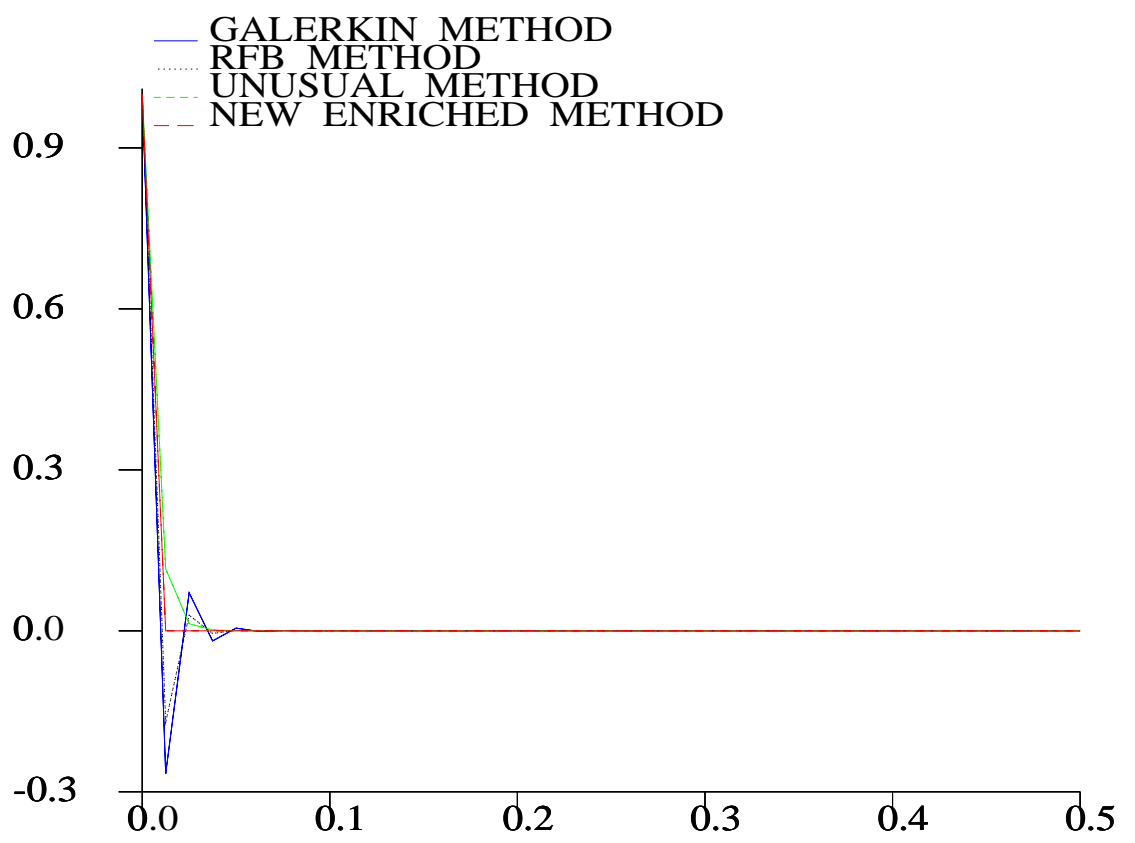

Figure 11. Profile of solutions at $x=0.5\left(\varepsilon=10^{-6}\right)$.

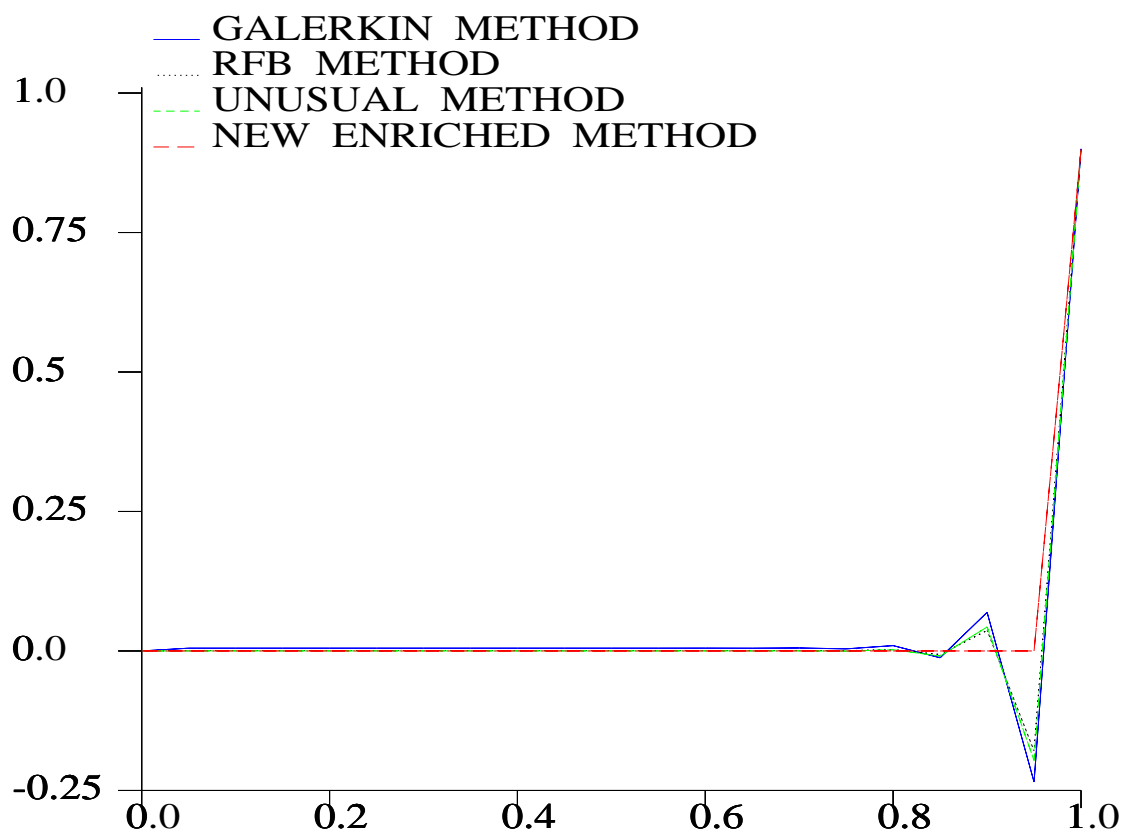

Figure 12. Profile of solutions at $y=0.05\left(\varepsilon=10^{-6}\right)$. 


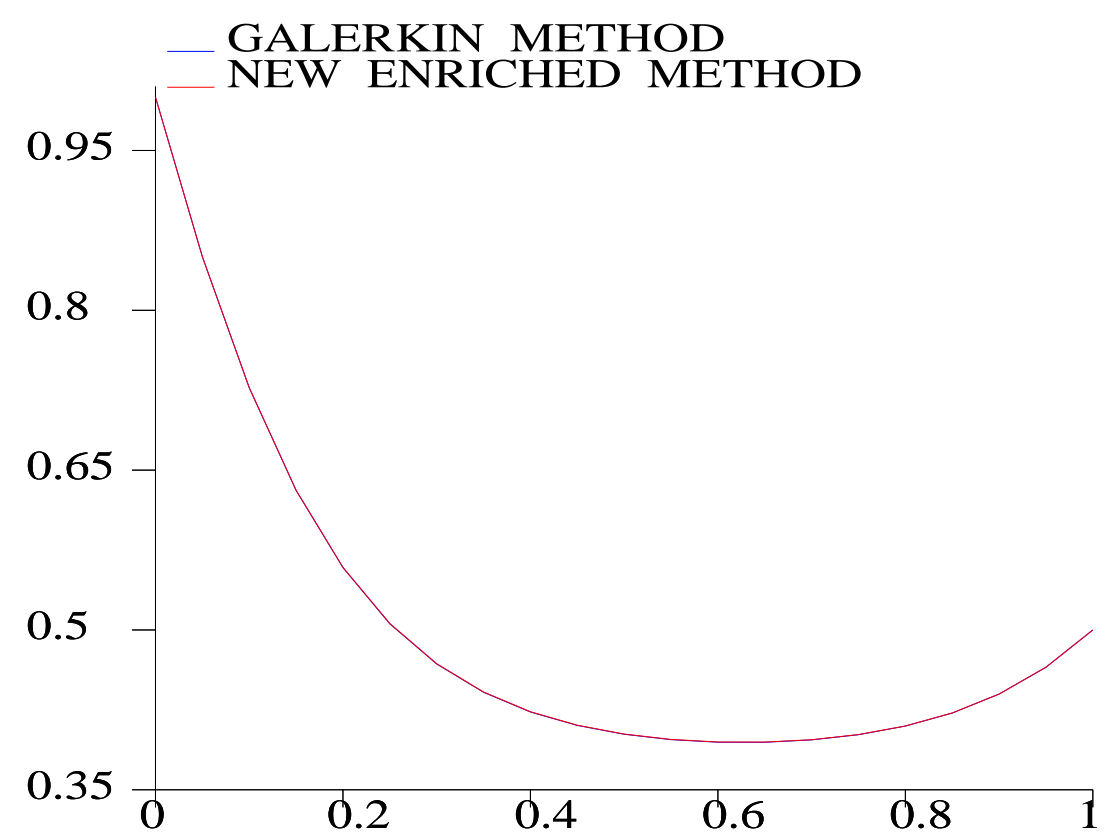

Figure 13. Profile of solutions at $y=0.5(\varepsilon=1)$.

\subsection{NACA problem.}

Here we illustrate the good performance of the present method even for unstructured meshes. We consider the domain $\Omega$ and its discretization as depicted in Figure 14 . We assume $f=0$ and homogeneous boundary Dirichlet conditions at the outer boundary. On the inner boundary we impose $u=1$. In all examples below we assume $\sigma=1$. In figure 15 we show that for moderate $\varepsilon$, both Galerkin and the present method perform well. As expected, for small $\varepsilon$, the Galerkin method presents spurious oscillations. It is remarkable here that even the unusual method is oscillatory, as shown in Figures 15, 17. As we show in Figure 16, the present method captures the boundary layer without any oscillatory behavior. 


\section{MESH\%anticlipinit}

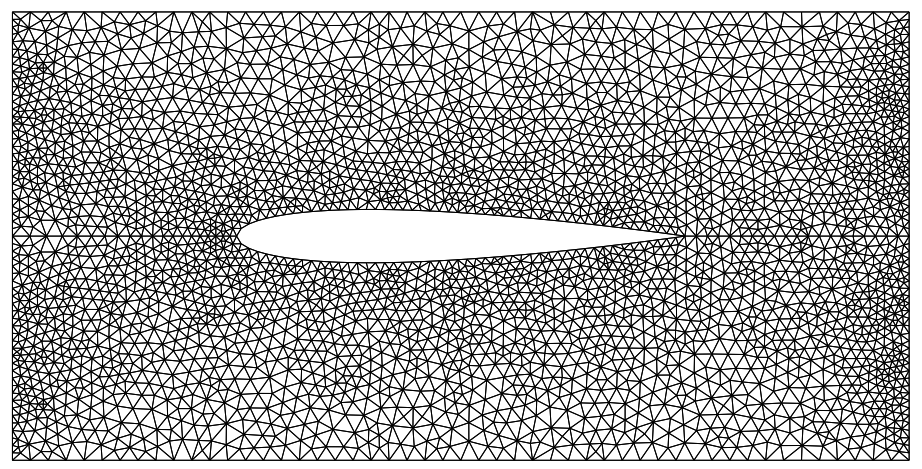

GALERKIN METHOD

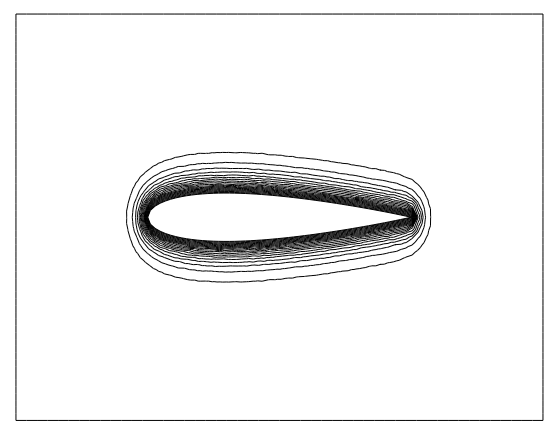

NEW ENRICHED METHOD\%anticlipinit

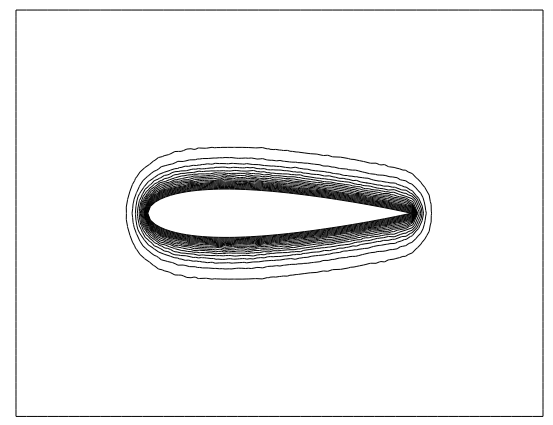

Figure 14. Isovalues of the solutions by Galerkin Method and by enriched method. Here $\varepsilon=10^{-2}$. 


\section{GALERKIN METHOD\%anticlipinit}

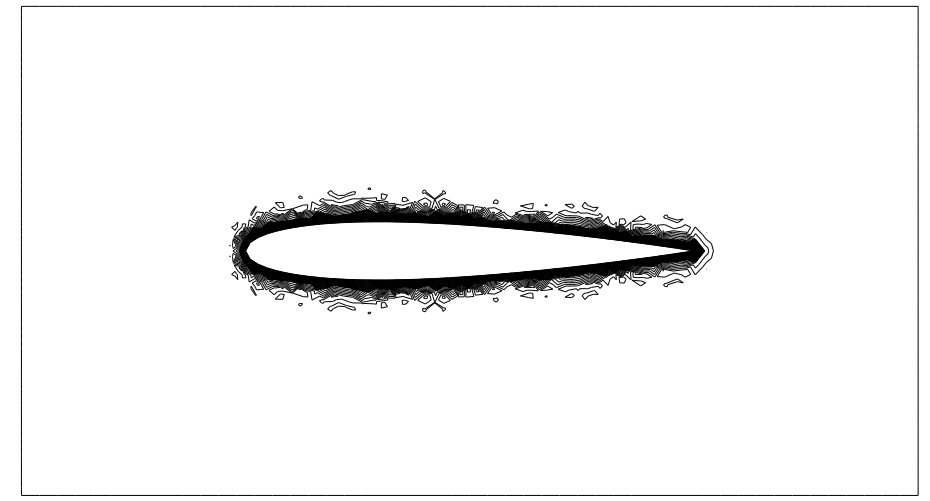

UNUSUAL METHOD\%anticlipinit

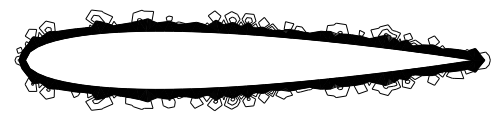

NEW ENRICHED METHOD\%anticlipinit

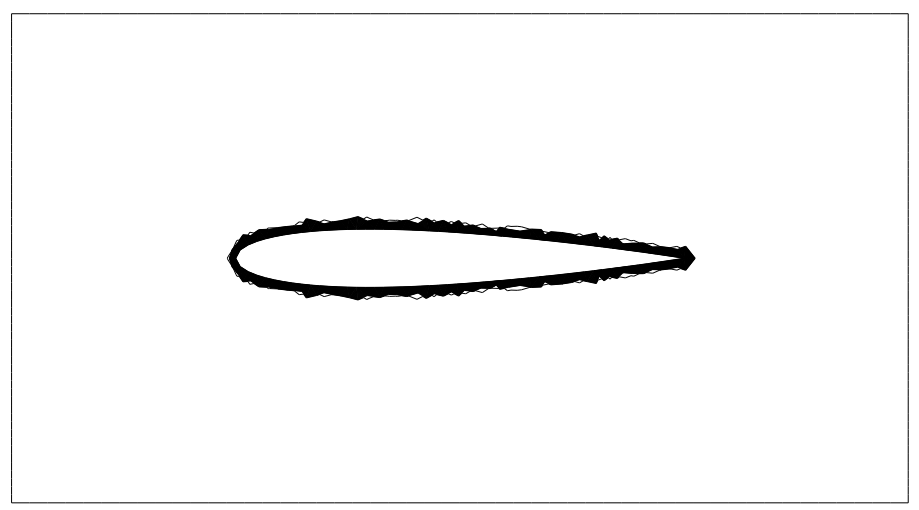




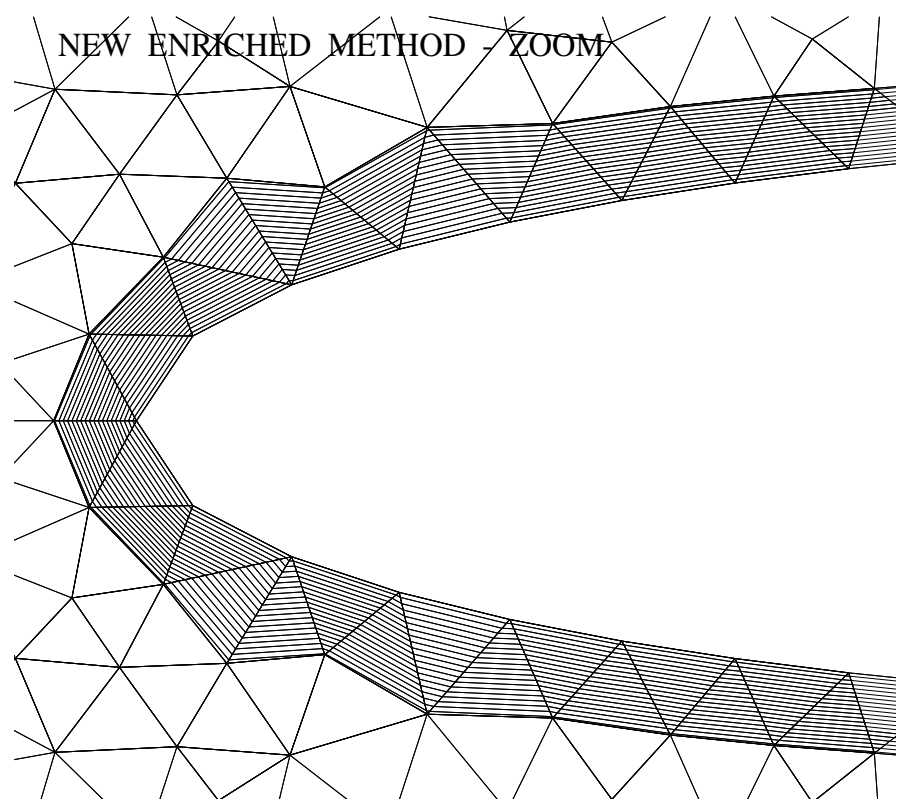

Figure 16. The new method captures the boundary layer accurately. Zoom of isovalues $\left(\varepsilon=10^{-6}\right)$.

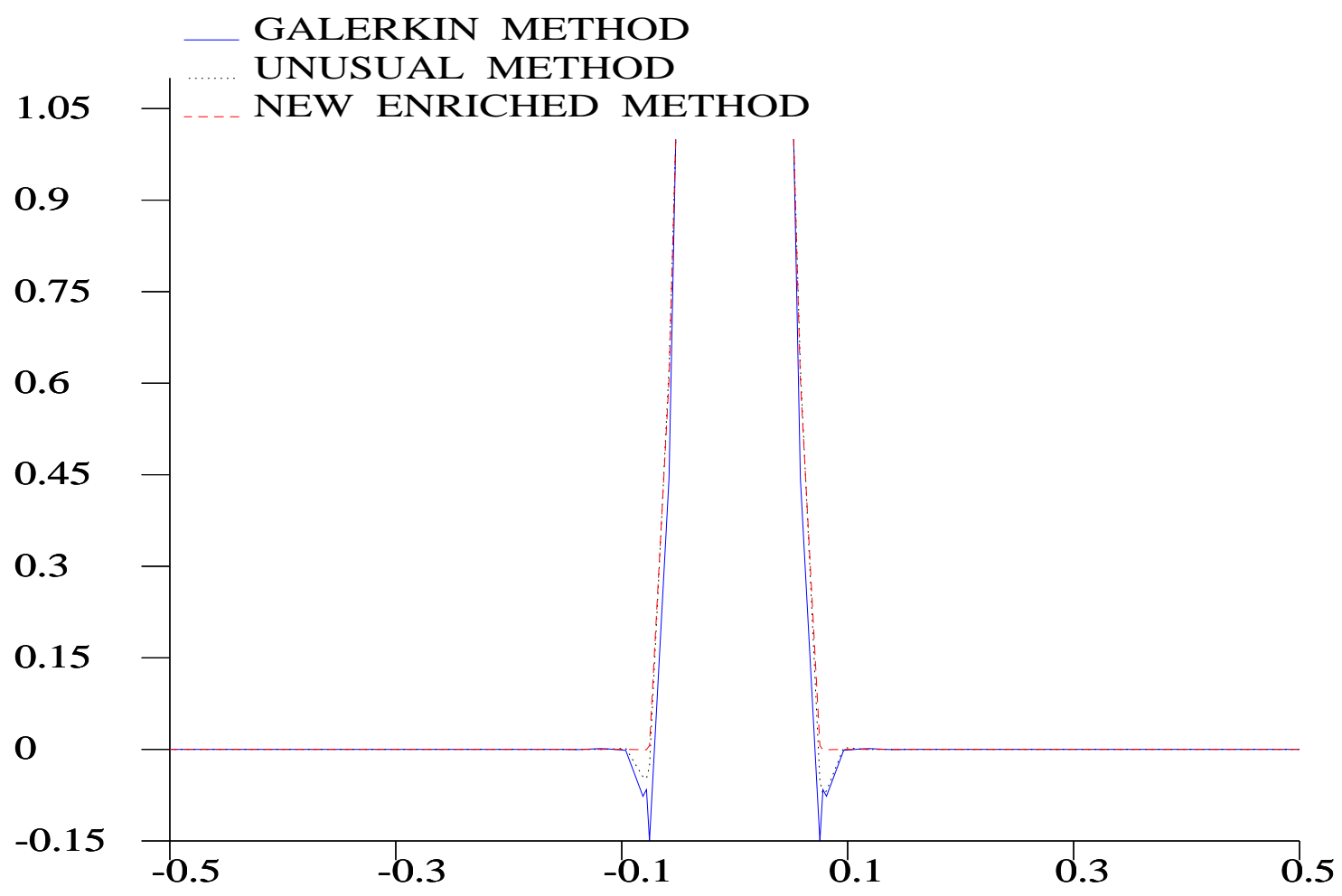

Figure 17. Profile of solutions at $x=0.5\left(\varepsilon=10^{-6}\right)$. 
Acknowledgments. This research was done while L. Franca was visiting the Applied Mathematics Department of LNCC. This author expresses his gratitude towards the colleagues of this Department for their hospitality. The first author was partially supported by CNPq-Brazil. The second and third authors were supported by CNPq-Brazil and FAPERJ-Brazil.

\section{REFERENCES}

[1] C. Baiocchi, F. Brezzi, And L. P. Franca, Virtual bubbles and the Galerkin-least-squares method, Comput. Meth. Appl. Mech. Engrg, (1993), pp. 125-141.

[2] G. Barrenechea And F. VAlentin, An unusual stabilized finite element method for a generalized Stokes problem, Numer. Math., (2002), pp. 653-677.

[3] F. Brezzi, M. O. Bristeau, L. P. Franca, M. Mallet, And G. Rogé, A relationship between stabilized finite element methods and the Galerkin method with bubble functions, Comput. Meth. Appl. Mech. Engrg., 96 (1992), pp. 117-129.

[4] F. Brezzi, L. P. Franca, T. J. R. Hughes, and A. Russo, $b=\int g$, Comput. Meth. Appl. Mech. Engrg., 145 (1997), pp. 329-339.

[5] F. Brezzi, L. P. FrancA, And A. Russo, Further considerations on residual-free bubbles for advectivediffusive equations, Comput. Meth. Appl. Mech. Engrg., 166 (1998).

[6] F. Brezzi And A. Russo, Choosing bubbles for advection-diffusion problems, Math. Models Methods Appl. Sci., 4 (1994), pp. 571-587.

[7] L. Franca, A. Madureira, L. Tobiska, and F. Valentin, Convergence analysis of a multiscale finite element method for singularly perturbed problems, preprint, (2003).

[8] L. P. Franca and E. G. Dutra do Carmo, The Galerkin gradient least-squares method, Comput. Meth. Appl. Mech. Engrg., 74 (1989), pp. 41-54.

[9] L. P. FRANCA AND C. FARHAT, Bubble functions prompt unusual stabilized finite element methods, Comput. Meth. Appl. Mech. Engrg., 123 (1995), pp. 299-308.

[10] L. P. Franca, C. Farhat, A. P. Macedo, And M. Lesoinne, Residual-free bubbles for the Helmholtz equation, Internat. J. Numer. Methods Engrg., 40 (1997), pp. 4003-4009.

[11] L. P. Franca And A. Russo, Approximation of the Stokes problem by residual-free macro bubbles, East-West J. Numer. Math., 4 (1996), pp. 265-278.

[12] — Deriving upwinding, mass lumping and selective reduced integration by residual-free bubbles, Appl. Math. Lett., 9 (1996), pp. 83-88.

[13] L. P. FRAncA And A. Russo, Mass lumping emanating from residual-free bubbles, Comput. Meth. Appl. Mech. Engrg., 142 (1997), pp. 353-360.

[14] — Unlocking with residual-free bubbles, Comput. Meth. Appl. Mech. Engrg., 142 (1997), pp. $361-364$.

[15] L. P. FRANCA AND F. VAlEntin, On an improved unusual stabilized finite element method for the advective-reactive-diffusive equation, Comput. Meth. Appl. Mech. Engrg., 190 (2000), pp. 1785-1800.

[16] I. HARARI AND T. J. R. Hughes, Stabilized finite elements methods for steady advection-diffusion with production, Comput. Meth. Appl. Mech. Engrg., (1994), pp. 165-191.

[17] T. Y. Hou AND X.-H. Wu, A multiscale finite element method for elliptic problems in composite materials and porous media., J. Comput. Phys., 134 (1997), pp. 169-189. 
[18] T. Y. Hou, X.-H. Wu, AND Z. CAI, Convergence of a multiscale finite element method for elliptic problems with rapidly oscillating coefficients, Math. Comp., 68 (1999), pp. 913-943.

[19] H. Roos, M. Stynes, And L. Tobiska, Numerical methods for singularly pertubed differential equations, Springer, 1991.

[20] A. Russo, Residual free bubbles and stabilized methods, in Proceedings of the Ninth International Conference on Finite Elements in Fluids - New Trends and Applications, M. M. Cecchi, K. Morgan, J. Periaux, B. A. Schrefler, and O. C. Zienkiewicz, eds., Venice, Italy, October 1995, pp. 1607-1615.

[21] F. Valentin And L. P. Franca, Combining stabilized finite element methods, Computational and Applied Mathematics, 14 (1995), pp. 285-300.

Department of Mathematics, University of Colorado at Denver, P.O. Box 17364, Campus Box 170, Denver, CO 80217-3364, USA

E-mail address: lfranca@math.cudenver.edu

Department of Applied Mathematics, Laboratório Nacional de Computação Científica, Av. Getúlio Vargas, 333, 25651-070 Petrópolis - RJ, Brazil

E-mail address: alm@lncc.br

Department of Applied Mathematics, Laboratório Nacional de Computação Científica, Av. Getúlio Vargas, 333, 25651-070 Petrópolis - RJ, Brazil

E-mail address: valentin@lncc.br 